\title{
Some Comments On: A Historical Note On The Proof Of The Area Of A Circle
}

Jaideep T. Naidu, Philadelphia University, USA

John F. Sanford, Philadelphia University, USA

\begin{abstract}
In a recent paper by Wilamowsky et al. [6], an intuitive proof of the area of the circle dating back to the twelfth century was presented. They discuss challenges made to this proof and offer simple rebuttals to these challenges. The alternative solution presented by them is simple and elegant and can be explained rather easily to non-mathematics majors. As business school faculty ourselves, we are in agreement with the authors. Our article is motivated by them and we present yet another alternative method. While we do not make an argument that our proposed method is any simpler, we do feel it may be easier to communicate to business school students. In addition, we present a solution using a rectangle which could be left as an exercise for the student after a brief explanation in class. Finding the area of a stack of rectangles with a rectangle as a starting point may seem redundant at first. However, we show that it is actually an excellent algebraic exercise for students since it offers a certain challenge which a square does not. We also solve this exercise using the quicker triangular approach and feel it can be appreciated by students in an Introduction to Calculus course. We also provide two interesting links that demonstrate the work of the ancient mathematicians for this well known problem.
\end{abstract}

Keywords: Area; Circle; History Of Mathematics; Pedagogy

\section{INTRODUCTION}

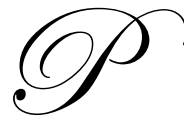

roofs that the area of a circle is $\Pi r^{2}$ have its origins from the time of the Greeks. Archimedes is credited to have provided the early proof for the area of a circle by dividing the circle into wedges and then fitting the wedges together in a way to approximate the rectangle (see e.g. [2]). Another popular historical version is that Archimedes is said to have divided the circle into an $n$-sided polygon where, as $n$ becomes larger and larger, the area of the polygon gets closer and closer to that of the actual circle. This is demonstrated at the end of the following link: http://www.ugrad.math.ubc.ca/coursedoc/math101/notes/integration/archimedes.html. Later proofs relied on calculus and infinite sequences which are generally difficult to communicate to nonmathematics majors. Epstein and Hochberg [3] presented an interesting and intuitive proof which was introduced in the twelfth century by Tosafot, a group of medieval rabbis. Garber and Tsaban [4] credit the proof to the $12^{\text {th }}$ century mathematician, Rabbi Abraham bar Hiya (RABH). His proof is basically about dividing a circle of radius $r$ into $n-1$ concentric washers of equal width and a circle in the middle with radius $\mathrm{r} / \mathrm{n}$. Considering the circle in the middle as a washer with inner circle radius zero, it can be viewed as a system of $n$ washers. The proof is about spreading out each of the washers (starting with the outermost one) so that it becomes flat. Its shape will be a trapezoid whose lower base equals the circumference of the outer washer, i.e., $2 \Pi r$, and whose upper base equals the circumference of the second outermost washer, i.e., $2 \Pi(\mathrm{r}-\mathrm{r} / \mathrm{n})$. Similarly, spread the next washer so that it lies right above the previous. Its lower base will be the same length as the upper base of the previous one. This process is continued until all $\mathrm{n}$ washers have been opened up. The area of the original circle is equal to the sum of the areas of all the trapezoidal figures. As $\mathrm{n}$ becomes large, the height of each trapezoid gets small and the entire figure becomes a triangle with base $2 \Pi r$ and with height $r$. Thus, says Tosafot, the area of the triangle is $(2 \Pi r) r / 2=\Pi r^{2}$. The following link provides a neat demonstration of his proof when you move the scroll bar to the right in the second picture.

http://www.cut-the-knot.org/Curriculum/Geometry/RABH.shtml 
This proof was challenged by later mathematicians like Bleicher [1] and Chavas Yair [5] and parallel solutions using squares were given instead. For details on these challenges and arguments, refer Wilamowsky et al. [6]. Our paper is motivated by Wilamowsky et al. [6] who offer a simple and elegant solution that can be comprehended by unsophisticated non-mathematics majors. Their main contribution was to offer a solution to the challenges by developing a different way to make the square washers open up. This is made possible by making the cuts in the corners of the square washers as seen in Diagram 1. This results in a stack of rectangles as shown in Diagram 2. Please refer their paper [6] for details on the mathematics. This resolves the issue of the space created by the wedges that were suggested by the earlier mathematicians.

Diagram 1

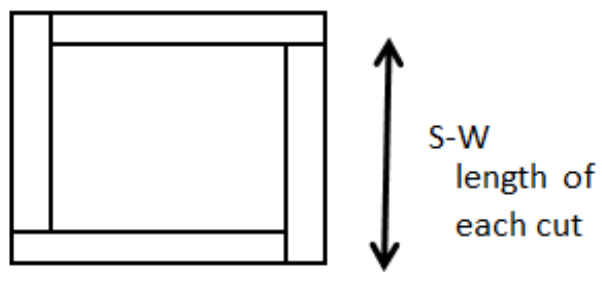

Diagram 2

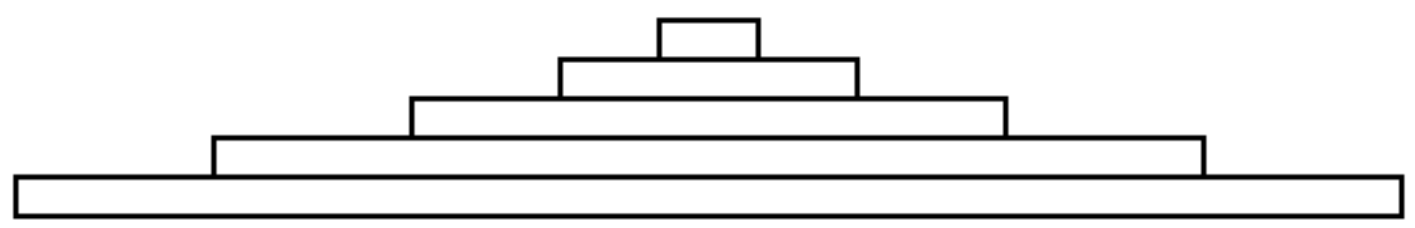

\section{OUR PROPOSED ALTERNATIVE SOLUTION}

As indicated earlier, our paper is motivated by the recent paper by Wilamowsky et al. [6]. We suggest the following cuts (Diagram 3) as shown below. Let "s" be the side of the square and "w" be the width of each washer. Note that $\mathrm{w}=\mathrm{s} / 2 \mathrm{n}$ where $\mathrm{n}$ is simply the number of washers formed out of the given square. Based on these cuts, the outermost washer is broken down into 4 pieces where 2 pieces have lengths $=\mathrm{s}$ units each and the other two pieces have lengths $=\mathrm{s}-2 \mathrm{w}$ units each. Now, $\mathrm{s}-2 \mathrm{w}$ is the length of the next outermost washer which would again be broken into 4 pieces: 2 pieces will have lengths $=\mathrm{s}-2 \mathrm{w}$ each and the other two pieces will have lengths $=\mathrm{s}-4 \mathrm{w}$ each and so on.

When the four pieces of the outermost washer are placed adjacent to each other, the result is a rectangle with length: $2 s+2(s-2 w)=4 s-4 w$. Similarly, the length of the rectangle formed by the next outermost washer is: $2(s-2 w)+2(s-4 w)=4 s-12 w$. Note that all the rectangles so formed will have the same width $w$.

The sum of the areas of the $n$ rectangles, for any value $n$, is

$w[4 s-4 w+\underline{4 s-12 w}+\ldots .+\underline{4 s-4 w(2 n-1)}]=w\left[4 s n-4 w(1+3+\ldots .+(2 n-1)]=w\left[4 s n-4 w n^{2}\right]\right.$

Substituting $w=s / 2 n$, the above equation is: $s / 2 n\left[4 s n-4(s / 2 n) n^{2}\right] \Rightarrow 2 s^{2}-s^{2}=s^{2}$. This is same as the result obtained by the authors. However, this may arguably be easier to communicate to non-mathematics majors.

In the next section, we present an exercise for the students. We believe this is an excellent algebra exercise to the non-mathematics major. This is an interesting problem because the length of a rectangle is larger than its 
breadth which results in the rectangular washers not having the same width on all four sides. This aspect is unlike all earlier examples proposed by the early mathematicians as well as in recent papers.

\section{Diagram 3}

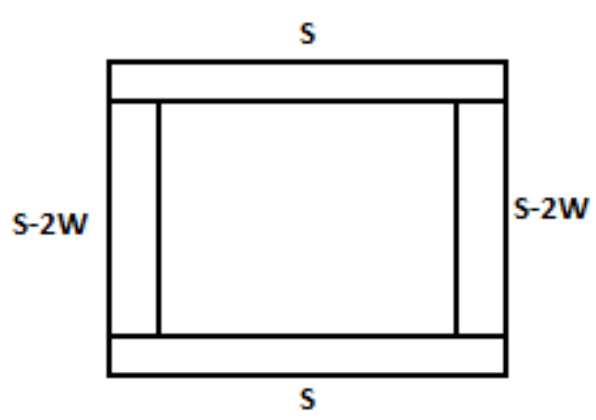

\section{A STUDENT EXERCISE}

Use a rectangle as the starting point and show all your work to obtain the sum of the areas of the $\boldsymbol{n}$ washers.

This exercise is to start with a rectangle and divide it into " $\mathrm{n}$ " rectangular shaped washers. As an example, consider a rectangle with length $(1)=150 \mathrm{~mm}$; and breadth $=90 \mathrm{~mm}$. Consider the following diagram (Diagram 4) with $\mathrm{n}=3$.

Diagram 4

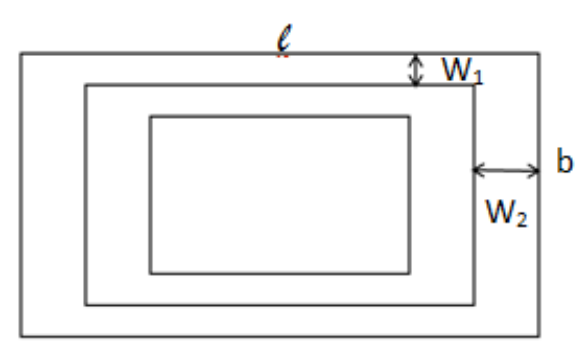

This means the outermost washer will have a width $=90 / 2 \mathrm{n}=90 / 6=15 \mathrm{~mm}$ on its longer sides and a width $=150 / 2 \mathrm{n}=150 / 6=25 \mathrm{~mm}$ on its shorter sides. Doing so will maintain the ratio of length:breadth for all the $\mathrm{n}$ rectangular washers. This is an interesting problem for the curious student because a logical way (and perhaps the only simple way) to solve such a problem is by stacking the longer and shorter pieces into two separate piles. This gives us two separate stacks of rectangles as shown in Diagram 5 below. The area of the original rectangle will now be the sum of these two stacks of rectangles. 
Diagram 5

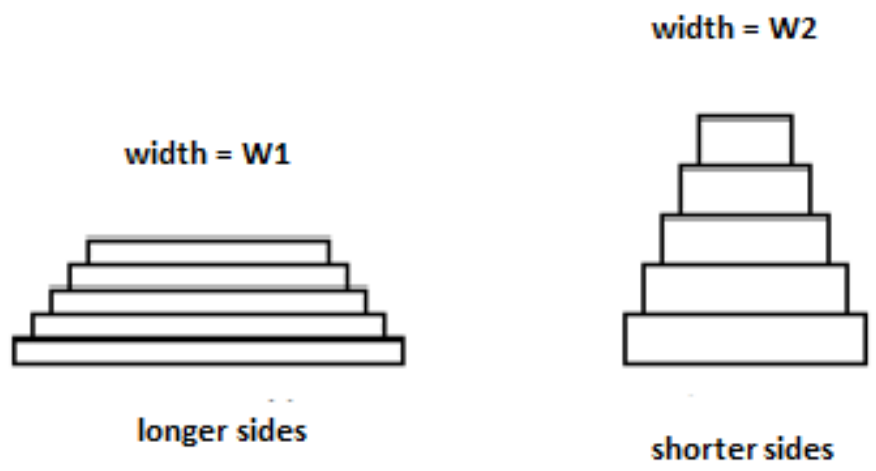

We use the same cuts as suggested by us earlier (and as shown in Diagram 6 below). Let "l" (length) be the longer side of the rectangle and "b" (breadth) be the shorter side of the side of the rectangle. Let $\mathrm{w}_{1}$ be the width of the longer side of the washer given by $b / 2 n$. Let $w_{2}$ be the width of the shorter side of the washer given by $1 / 2 n$. Note that $\mathrm{n}$ is simply the number of washers formed out of the given rectangle.

\section{Diagram 6}

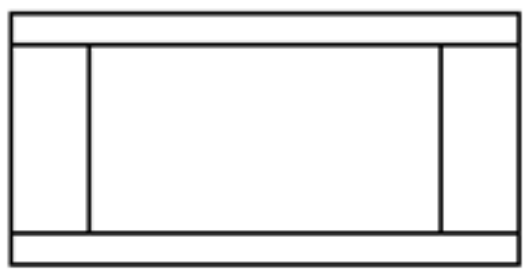

Based on these cuts, the outermost washer is broken down into 4 pieces where 2 pieces have lengths $=1$ units each and the other two pieces have lengths $=b-2 w_{1}$. Note that $1-2 w_{2}$ is the length of the next outermost washer which would again be broken into 4 pieces: 2 pieces will have lengths $=1-2 \mathrm{w}_{2}$ each and the other two pieces will have lengths $=\mathrm{b}-4 \mathrm{w}_{1}$ each and so on.

The two longer pieces of the outermost rectangular washer are placed adjacent to each other and the resulting rectangle has a length $=21$. The length of the rectangle formed by the longer pieces of the next outermost washer will be $2\left(1-2 w_{2}\right)=2 l-4 w_{2}$ and so on. This is the first stack of the $n$ washers.

The second stack is formed by placing the shorter pieces of each washer in one pile. The length of the rectangle formed by the outermost washer in this case is $2\left(b-2 w_{1}\right)=2 b-4 w_{1}$. Similarly, the length of the rectangle formed by the two shorter pieces of the next outermost washer will be $2\left(b-4 w_{1}\right)=2 b-8 w_{1}$. This is the second stack of the $n$ washers.

These two stacks are as shown in Diagram 5 above. The objective is to find the sum of the areas of these two stacks of rectangles.

Area of Stack 1 (formed by the longer pieces of the $n$ washers):

$\mathrm{w}_{1}\left[\underline{2 \mathrm{l}}+\underline{2 \mathrm{l}-4 \mathrm{w}_{2}}+\underline{2 \mathrm{l}-8 \mathrm{w}_{2}}+\ldots \ldots+\underline{2 \mathrm{l}-4 \mathrm{w}_{2}} \underline{(\mathrm{n}-1)}\right]=\mathrm{w}_{1}\left[2 \ln -4 \mathrm{w}_{2}(1+2+\ldots+\mathrm{n}-1)\right]=$ $\mathrm{w}_{1}\left[2 \ln -4 \mathrm{w}_{2}(\mathrm{n}-1)(\mathrm{n}) / 2\right]$

Substituting the values of $\mathrm{w}_{1}$ and $\mathrm{w}_{2}$ and simplifying further results in an area $=(\mathbf{l b} / \mathbf{2})+(\mathbf{l b} / \mathbf{2 n})$. 
Area of Stack 2 (formed by the shorter pieces of the $n$ washers):

$\mathrm{w}_{2}\left[\underline{2 \mathrm{~b}-4 \mathrm{w}_{1}}+\underline{2 \mathrm{~b}-8 \mathrm{w}_{1}}+\underline{2 \mathrm{~b}-12 \mathrm{w}_{1}}+\ldots . \underline{2 \mathrm{~b}-4 \mathrm{w}_{1}}(1+2+\ldots+\mathrm{n})\right]=\mathrm{w}_{2}\left[2 \mathrm{bn}-4 \mathrm{w}_{1}(1+2+\ldots+\mathrm{n})\right]$

$=\mathrm{w}_{2}\left[2 \mathrm{bn}-4 \mathrm{w}_{1}(\mathrm{n})(\mathrm{n}+1) / 2\right]$

Substituting the values of $\mathrm{w}_{1}$ and $\mathrm{w}_{2}$ and simplifying further results in an area $=(\mathbf{l b} / \mathbf{2})-(\mathbf{l b} / \mathbf{2 n})$. Adding up the areas of these two rectangles results in an area $=\mathbf{l b}$ as expected.

\section{THE TRIANGULAR APPROACH FOR THE ABOVE STUDENT EXERCISE}

From Diagram 5, it is easy to see for a student with a good mathematical background that if $\mathrm{n}$ is very large (resulting in very small widths), the two stacks end up being triangles. The triangle with the larger base (= 2l) will have a smaller height $\left(=n w_{1}\right)$ and the one with the shorter base $\left(=2 b-4 w_{1}\right)$ will have a larger height $\left(=n w_{2}\right)$. Using the standard formula of a triangle (Base*Height/2), the area of the first triangle $=21 * \mathrm{nw}_{1} / 2=(21 * \mathrm{nb} / 2 \mathrm{n}) / 2=\mathrm{lb} / 2$; and the area of the second triangle $=\left(2 \mathrm{~b}-4 \mathrm{w}_{1}\right) * \mathrm{nw}_{2} / 2=(2 \mathrm{~b}-(4 \mathrm{~b} / 2 \mathrm{n})) *(\mathrm{nl} / 2 \mathrm{n}) / 2=(2 \mathrm{~b}-2 \mathrm{~b} / \mathrm{n}) *(\mathrm{nl} / 2 \mathrm{n}) / 2=2 \mathrm{~b}(1-$ $(1 / n) *(n 1 / 2 n) / 2$. As $n$ becomes very large, $1 / n$ becomes very small and can be assumed to be zero. Thus, the area of this triangle $=2 \mathrm{~b} *(\mathrm{nl} / 2 \mathrm{n}) / 2=\mathrm{lb} / 2$. Therefore the sum of areas of these two triangles $=\mathrm{lb}$.

\section{CONCLUSION}

We presented an alternative set of cuts to communicate the method proposed by Wilamowsky et al. [6]. In addition, we presented an interesting exercise for students. We believe that when the starting point is a rectangle, the algebra is more involved and can be a good practice exercise for students. We also solved this exercise using the triangle approach which is quick and easy and can be appreciated by some students. The links provided in this paper are interesting and can motivate the students to understand the concepts in Algebra or Introduction to Calculus courses.

\section{AUTHOR INFORMATION}

Jaideep Naidu is an Associate Professor of Operations Management and Management Science at the School of Business Administration, Philadelphia University. He holds a Ph.D. in Operations Management from The University of Mississippi. His research interests center on machine scheduling problems and higher education. Among other journals, his work has appeared in Journal of the Operational Research Society, Omega - The International Journal of Management Science, and International Journal of Operations and Quantitative Management. E-mail: naiduj@philau.edu

John Sanford is a Professor of Information Systems at the School of Business Administration, Philadelphia University. He received Doctor of Engineering degree from Yale University 1963. His interests include information systems, Data Analysis, and data Analysis with Fuzzy Systems. He has published in Proceedings of the Northeast Decision Sciences, Proceedings of IABPAD New Orleans, and The International Journal of Teaching \& Case Studies IJTCS. E-mail: sanfordj@philau.edu

\section{REFERENCES}

1. $\quad$ Bleicher, M., Maskil el David, Jerusalem, Israel, 1988. (In Hebrew)

2. Dunham, W. Archimedes' Determination of Circular Area. Ch. 4 in Journey through Genius: The Great Theorems of Mathematics, Wiley, New York, 1990, 84-112.

3. Epstein, S. and M. Hochberg, A Talmudic Approach to the Area of a Circle, Mathematics Magazine, September 1977, 210.

4. Garber, D. and B. Tsaban, A Mechanical Derivation of The Area of The Sphere, The American Mathematical Monthly, January 2001, 10-15.

5. Shalot Utshuvot Chavas Yair, Responsa 172, Jerusalem Edition, 1987 (in Hebrew).

6. Wilamowsky, Y., Epstein S. and B. Dickman, A Historical Note on The Proof of The Area Of A Circle, Journal of College Teaching and Learning, 8(3), 2011, 1-5. 
7. $\quad$ http://www.ugrad.math.ubc.ca/coursedoc/math101/notes/integration/archimedes.html

8. http://www.cut-the-knot.org/Curriculum/Geometry/RABH.shtml

\section{NOTES}

